\title{
Diseño de modelos complejos para la simulación de sistemas socio-técnicos*
}

\author{
Miguel Melgarejo ${ }^{1}$ \\ (D) http://orcid.org/0000-0003-3387-9931 \\ Universidad Distrital Francisco José de Caldas, Colombia \\ Nelson Obregón ${ }^{2}$ \\ (iD http://orcid.org/0000-0002-0881-9833 \\ Pontificia Universidad Javeriana, Colombia
}

DOI: http://dx.doi.org/10.17081/eduhum.19.33.2647

Recibido: 26 de octubre de 2016

Aceptado: 14 de abril de 2017

\section{Design of complex models for the simulation of socio-technical systems}

Palabras clave:

Sistemas socio-técnicos,

Sistemas multi-agentes,

Diseño, Ingeniería, Simulación.

Key words: Socio technical systems, Multiagent systems, Design, Engineering, Simulation.

\begin{abstract}
Resumen
En este artículo se desarrolla una reflexión sobre los sistemas socio-técnicos y su modelado desde la perspectiva de los sistemas complejos. Particularmente, la reflexión se centra en un marco de complejidad reciente y en la integración ser-mundo propuesta en la fenomenología. Así, la reflexión deja entrever una propuesta metodológica para la simulación de sistemas socio-técnicos basada en micro-mundos que se relacionan en macro-mundos. A manera de ilustración, se describe un modelo de simulación para el crimen urbano en el cual se considera que dicho fenómeno resulta de la dinámica de un sistema socio-técnico conformado por la ciudad y sus habitantes. Al final, se propone una discusión sobre posibilidades y perspectivas futuras frente a la reflexión desarrollada.
\end{abstract}

\begin{abstract}
In this article a reflection about socio-technical systems is developed, and is modeled from the perspective of complex systems. Particularly, the reflexion is centered in a framework of recent complexity and in the integration of being/world relationship proposed by phenomenology. Thus, the reflection makes a methodological proposal foreseeable for the simulation of socio technical systems based on microworlds that are related to micro worlds. By way of illustration, a simulation model for urban crime has been described, in which this phenomenon is considered as the result of a socio technical dynamics formed by the city and its inhabitants. At the end, a discussion is proposed about future possibilities and perspectives related to the reflections of the topic developed.
\end{abstract}

\section{(c) $(9) \odot$}

Referencia de este artículo (APA): Melgarejo, M. \& Obregón, N. (2017). Diseño de modelos complejos para la simulación de sistemas socio-técnicos. En Revista Educación y Humanismo, 19(33), 320-333. http://dx.doi.org/ 10.17081/eduhum.19.33.2647

\footnotetext{
* Artículo de reflexión vinculado al proyecto de investigación aportes a la caracterización y el modelado de la dinámica del crimen urbano desde la ingeniería de sistemas complejos.

1. IE, MIE, Dr. Ing (c), Pontificia Universidad Javeriana, Profesor Facultad de Ingeniería, Universidad Distrital Francisco José de Caldas, Bogotá DC.mmelgarejo@udistrital.edu.co

2. IC, M.Sc, Ph.D, Profesor Facultad de Ingeniería, Pontificia Universidad Javeriana, Bogotá DC. nobregon@javeriana.edu.co
} 


\section{Introducción}

Los sistemas socio-técnicos son aquellos donde interactúan seres humanos y componentes artificiales creados por estos mismos para llevar a cabo sus propósitos (Kroes, 2012; Kroes et al., 2006). Quizás una perspectiva más amplia de esta clase de sistemas debería incluir la relación de los seres humanos con su entorno natural. En ese sentido, el sistema socio-técnico estaría asociado no solo a un paisaje artificial sino también a un paisaje natural; sin embargo, definir la frontera donde uno comienza y el otro termina es algo difuso.

Los subsistemas que aparecen dentro de un sistema socio-técnico pueden tener o no un propósito y quizás la definición de ello estaría ligada a su origen desde el entorno puramente natural o el entorno artificial creado por el hombre (Ackoff \& Gharajedaghi 1961). Inclusive la interpretación que se pueda hacer del ser humano como partícipe de esta clase de sistemas sería ambigua, pues su condición humana no es algo completamente definido y, por el contrario, se adapta según las circunstancias y su relación con el mundo (Arendt, 1958). No se podría decir entonces que un sistema socio-técnico estaría caracterizado en su totalidad por la presencia de seres humanos en su calidad de animal laborans, homo faber, homo oeconomicus o cualquier otra etiqueta creada para definir su condición.

Tratar entonces de definir un sistema sociotécnico desde la pura perspectiva de los artefactos que lo componen facilitaría la aplicación de las leyes y teorías que rigen su comportamiento pero también eliminaría de plano todo el matiz humano que le da sentido. En cambio, buscar entender el sistema socio-técnico únicamente desde la dimensión humana, implica abordar primero una serie de preguntas sobre las cuales aún no tenemos respuestas claras: ¿Qué es el ser? ¿Qué es el mundo? ¿Cómo se relaciona el ser con el mundo?

Independientemente del problema de la definición, hoy en día se reconoce que los sistemas socio-técnicos exhiben algunas propiedades de interés que harían que estos sistemas fuesen catalogados como complejos. Algunas de estas propiedades son: presencia de no-linealidades, multiplicidad de interacciones, adaptabilidad, auto-organización, imprevisibilidad, incontrolabilidad, entre otras (Jelinek, Romme \& Boland, et al, 2008; Sice \& French, 2006). En ese sentido, la pregunta por el modelado de esta clase de sistemas desbordaría los supuestos de generalidad y universalidad propios de la ciencia (Kroes, 2012; Heidegger, 1938), pero al mismo tiempo posibilitaría que la concepción de tales modelos sea entendida desde otras formas de conocimiento (Heidegger, Simon, 1988). Es relevante insistir por ello en que los sistemas socio-técnicos no son estrictamente tales, pero tienen un alto grado de artificialidad que los hace contingentes. Tratar de modelarlos implica una interpretación que, dado el caso, puede llegar a perturbarlos o modificarlos (Kroes et al., 2006; Jelinek et al., 2008).

La reflexión que se desarrolla en este artículo 
se aproxima a la pregunta por el modelado de sistemas socio-técnicos desde la ingeniería y el diseño, partiendo de las siguientes consideraciones:

- Los sistemas socio-técnicos exhiben rasgos de sistemas complejos, por tanto, la pregunta por el diseño de sus modelos de simulación debería considerar la construcción de estos últimos sistemas.

- La construcción de modelos complejos para sistemas socio-técnicos se puede desarrollar por medio de bloques constructores dinámicamente relacionados.

- Los bloques constructores podrían diseñarse desde la cotidianidad del ser y la relación con su mundo. En ese sentido, la psicología cognitiva puede enlazarse con los sistemas de múltiples agentes para aproximarse a tal entendimiento.

Tras la introducción antes planteada, el presente artículo se desarrolla de la siguiente manera: la sección II muestra un marco referencial sobre el cual se entenderá la complejidad de un sistema socio-técnico. Igualmente, ese punto se pretende ver desde la práctica, porque sería necesario que un modelo de simulación de un sistema complejo sea en sí mismo un sistema complejo. Luego, en la sección III se encuentra una propuesta de diseño basada en bloques constructores, sistemas de múltiples agentes y ciencia del conocimiento. En la sección IV se describirá un caso de aplicación enfocado en el crimen urbano, el cual viene siendo tratado por los autores. Esta misma sección aborda una discusión respecto al aprendizaje y educación en cuanto al modelado de sistemas socio-técnicos. Finalmente, en la sección V, se señalan algunas conclusiones.

\section{Modelos complejos para sistemas complejos}

\section{Un marco de complejidad}

La complejidad de un sistema es un concepto considerado hoy en día un tanto amplio y puede variar según el contexto desde el cual se estudie. En este caso se tendrá en cuenta un marco de complejidad recientemente descrito por Arnold (2013), que no se enfoca en proveer una definición del término complejidad, sino en tipificar los sistemas de acuerdo con su estructura y el conocimiento necesario para abordar su gestión. El concepto de gestión se entiende como la posibilidad de afectar el sistema, más que de controlarlo. Así, en la tipificación propuesta, los sistemas socio-técnicos se encuentran en el nivel más alto de complejidad:

Los sistemas complejos tipo I se caracterizan por estar gobernados por reglas simples y exhiben comportamientos complejos como oscilaciones irregulares, atractores caóticos, auto-organización y emergencia. Los modelos de estos sistemas pueden ser expresados en términos de sistemas auto-regresivos, ecuaciones diferenciales no-lineales y flujos de información, entre otras herramientas. Un ejemplo de esta clase de sistemas son las placas tectónicas, en el caso de sistemas naturales, o el juego de la vida de Conway, en el caso de artefactos creados por el hombre (Bak, 1991). 
Los sistemas complejos tipo II contienen multiplicidad de reglas y procesos interactuantes. Se caracterizan por exhibir diferentes comportamientos en diferentes escalas. En este caso los modelos no solo deben capturar las reglas sino también las transiciones de escala. Los modelos de estos sistemas pueden ser cualitativos (ej. Mapas mentales), semi-cuantitativos (ej. Ecuaciones diferenciales difusas) y cuantitativos (ej. Modelamiento integrado). Un ejemplo de esta clase de sistema son los procesos de deforestación, debido al cambio del uso de la tierra. Se podría decir que en este nivel de complejidad la actividad humana ya se hace evidente.

Cuando los sistemas requieren gestionar diversas perspectivas con el fin de establecer unos objetivos comunes, hacen parte de los sistemas complejos tipo III. En esta clase de sistema los actores humanos (por ejemplo stakeholders) se hacen evidentes y participan de forma activa, interpretando de forma diferente la dinámica del sistema. Usualmente tales actores establecen compromisos que tienden a ser subjetivos e influenciados por la cultura. Estos sistemas se han intentado modelar desde la teoría de la decisión y la optimización multicriterio, entre otros.

La complejidad tipo $I V$ se da en el caso de estructuras institucionales creadas por las sociedades para gestionar. En estos sistemas aparecen múltiples actores como: los gobiernos nacionales, la industria, los gobiernos locales o la academia. Algunos de los individuos involucrados en estos tratan de ejecutar tácticas para lograr objetivos de corto o largo plazo.
La complejidad de los sistemas socio-técnicos podría enmarcarse en los tipos III y IV, pues, se caracterizan por una adaptación continua que conlleva a la imprevisibilidad e incontrolabilidad de su comportamiento. En cuanto al modelamiento de estos sistemas se refiere, la dinámica de sistemas se muestra como una herramienta prometedora (Schwaninger, Olaya, \& Ambroz, 2007). En ese sentido, en su creación resulta necesaria la participación de múltiples formas de conocimiento, de manera que se trascienda la visión puramente teórica y científica (Gadamer, 1960).

\section{Modelos complejos desde la práctica}

El modelado de sistemas complejos exige hoy en día un ejercicio que trasciende el reduccionismo característico de la ciencia, ya que en estos se reconoce que el todo es más que la suma de las partes y estas no son necesariamente las explicaciones más sencillas y probadas bajo las mismas condiciones, aunque sean las correctas (Mendel, 2007). La práctica del modelado de sistemas complejos, incluidos los sistemas socio-técnicos, busca trasladar algo de las propiedades del sistema real a una representación artificial. El propósito de ese movimiento de lo real a lo artificial dependerá de la intención del modelador. De este modo, en algunos casos, se tratará de aproximarse a una predicción; en otros, de buscar una explicación, formular una pregunta o iniciar un debate (Pahl-Wostl, 2007; Epstein, 2008).

Los practicantes del modelado de sistemas 
socio-técnicos vienen reconociendo que los modelos de simulación de sistemas complejos deberían exhibir los mismos atributos de tales sistemas. Por ejemplo, luego de una amplia aplicación de sistemas multi-agentes para la gestión de sistemas socio-técnicos, Zevski (2012) afirma: "Solo los modelos complejos se pueden utilizar para representar sistemas complejos" (Zevski, G, 2012, p.434). Así, cuando se trata de modelar un sistema complejo este puede sufrir adaptaciones y cambiar, por tanto, los cambios que se produzcan deberían trasladarse al modelo. Esto solo es posible si el modelo es plástico o adaptable, pero sería igual de interesante que el modelo tuviera la capacidad de introducir los cambios autónomamente, lo cual se hace viable si tiene capacidad de auto-organización.

Una línea similar de pensamiento se puede ver en los sistemas de soporte de la decisión. Jakeman, quien ha desarrollado esta clase de sistemas para la planeación de políticas, indica (Jakeman et al, 2011, p. 272): "Para los modelos integrados, existen principios similares a los de los modelos individuales: la reducción de la complejidad sin omitir componentes cruciales es en muchos casos la mejor solución. Sin embargo, el sistema final podría ser bastante complejo, ya que el sistema del mundo real es intrínsecamente complejo" (p. 272).

Aquí se evidencia que debería existir un modelo mínimo que aborde la complejidad del sistema real. En tal caso, los componentes cruciales deben ser identificados y el modelo deberá preservarlos. Introducir otros componentes, más allá de los componentes esenciales, implica correr procesos de modelado más detallados que no necesariamente mejoran el entendimiento del comportamiento del modelo, pero sí incrementan su costo computacional y de mantenimiento. Los modelos más sencillos se pueden utilizar para guiar las sesiones de discusión (por ejemplo workshops), mientras que los modelos más detallados se reservan para un análisis profundo de la dinámica del sistema.

La perspectiva de integrar la complejidad en los modelos de simulación con un costo razonable en esfuerzo, tiempo y recursos se hace presente desde el punto de vista de otros practicantes y áreas de conocimiento. Por citar algunos ejemplos, en Berger et al. (2007), se emplea tecnología de sistemas de múltiples agentes para capturar la complejidad del problema del uso del agua y del comportamiento social de los usuarios; en Parrott (2011), se considera el uso de modelos híbridos (por ejemplo sistemas multiagentes interactuando con otras tecnologías) para integrar en una simulación la complejidad de sistemas sociales y biofísicos que interactúan como mecanismo de soporte para la toma de decisiones. Igualmente, en Torrens y Nara (2007), se consideran modelos híbridos compuestos por sistemas multi-agentes y autómatas celulares para estudiar el problema de la gentrificación urbana.

\section{Diseño de modelos complejos para sistemas} socio-técnicos

\section{Diseño a partir de bloques constructores}

El proceso de diseño de un sistema es un proceso de conocimiento-creación dirigido por el aprendizaje. En este sentido, Cowan, Allen \& 
Mistree (2006, p.371) proponen una metáfora para el diseño de sistemas complejos:

De manera similar a las interacciones de los niños con los micromundos educativos, imaginamos la creación y exploración de micromundos por parte de profesionales dedicados a diseñar sistemas de ingeniería complejos... los diferentes grupos involucrados en el diseño colaborativo deben ser capaces de modelar, explorar $\mathrm{y}$, en última instancia, integrar micromundos diferentes. Para ello, empleamos la siguiente analogía: al igual que los niños construyen castillos con bloques de construcción de juguete, los diseñadores construyen micromundos con bloques de construcción de diseño...

Se puede entonces entender el proceso de diseño de un sistema complejo como un acto de creación en el cual se involucran diferentes bloques constructores. El sistema complejo en sí sería un macro-mundo compuesto por una serie de micro-mundos conformados por los bloques constructores. Tanto los bloques constructores como los micro-mundos se encuentran dinámicamente relacionados. En ese sentido, un modelo de simulación de un sistema sociotécnico podría construirse desde esta perspectiva si se le considera como un sistema complejo en sí mismo. Así, los micro-mundos de simulación se relacionarían entre sí intercambiando información con el fin de conformar macro-mundos de simulación sobre los cuales se delinearía el modelo del sistema socio-técnico. Evidentemente, esta perspectiva encierra una gran cantidad de elementos similares a los que se pueden observar desde la dinámica de sistemas (Schwaninger et al., 2007) y, de hecho, debería beneficiarse de toda la base conceptual común que exista, sin embargo, el concepto de micromundo define un elemento particular y diferenciador.

En el caso de los sistemas socio-técnicos, los bloques constructores deberían apuntar al modelamiento de agentes interactuantes que reflejen la presencia de seres humanos. Los micro-mundos se concebirían como redes de agentes o reglas que pueden ser definidas por el diseñador desde un principio. También sería interesante dejar campo a que tales redes se formaran como resultado de procesos de auto-organización. Asignar significado a estos mundos, en este contexto, se hace más difuso, no obstante, estos podrían representar asociaciones de humanos y artefactos, pequeñas organizaciones o territorios operantes en escalas de tiempo relativamente pequeñas. Los macromundos se conformarían como la interconexión de mundos más pequeños, reflejando la estructura de organizaciones más grandes que se nutren de la dinámica de pequeñas organizaciones. Se esperaría que los macro-mundos operaran en escalas de tiempo diferentes. Se podría pensar que su conformación sea labor del diseñador del modelo o resultado de procesos de auto-organización. Bajo esta perspectiva, el diseño de un modelo tendría que posibilitar procesos de autoorganización en diferentes escalas.

\section{Bloques constructores y agentes}

Los bloques constructores deben entenderse desde el concepto de agente. En este contexto, 
los agentes se conciben como elementos de acción que representan seres humanos. Dada su autonomía, plasticidad y apertura a la posibilidad de ser, establecer una teoría rectora de los comportamientos humanos es una empresa aún en desarrollo, cuyo final no está nada cercano (Jelinek, et al., 2008). De hecho, la forma como los seres humanos se relacionan con los objetos tecnológicos que estos mismos fabrican está condicionada por su autonomía (Arendt, 1958). Al final, cada individuo puede hacer una libre interpretación de los objetos que forman parte de su mundo, por lo que prever las consecuencias de la relación individuo-tecnología podría escapar a cualquier esfuerzo intelectual.

A pesar de que quizás sea infructuoso tratar de capturar la complejidad de un ser humano en un agente, ello no significaa que esta tarea no se deba abordar desde algún punto de vista. Pero es necesario trascender la esfera científica para emprender esta acción. La ingeniería y el diseño de sistemas de múltiples agentes pueden encontrar focos de inspiración en diferentes representaciones del mundo (Peñaloza, 2015) $\mathrm{y}$, en ese sentido, se han venido dando algunos pasos desde la psicología filosófica, sin embargo el carácter científico (por ejemplo, ciencia del conocimiento) que se le da al tratamiento del problema aún es latente (Dreyfus, 2003; Sun, 2001).

Uno de los aspectos críticos en el diseño de agentes integrando una perspectiva que considere al ser humano es el problema de la interacción. Es característico del ser humano y de sus organizaciones que su interacción se base en procesos de comunicación (Jelinek et al. 2008). Efectivamente, tan inmerso está el hombre en su comunicación con los otros que esta forma parte de su cotidianidad, y la comunicación requiere de un fondo común en el que los agentes comparten tanto su mundo como su interpretación en términos de reglas. En el diseño basado en bloques constructores, se podría pensar que los agentes asociados en un mismo micro-mundo podrían satisfacer esa condición, dada como un requisito de diseño a priori. De este modo, un grupo de agentes puede compartir el mismo ambiente, expresado en términos de objetos y relaciones entre estos, sin embargo, cada agente puede interpretar tal contexto de una forma diferente, de manera tal que su mundo adquiere una dimensión particular. Así el agente y su mundo formarían una unidad, generándose no solo una interacción entre agentes sino también de mundos, sobre la base de un contexto común.

El micro-mundo conformado por los agentes humanos debería considerar la noción de un campo. Es decir, un espacio sobre el cual se pueda construir una estructura social estable, en la que los agentes puedan interpretar distintos roles en diferentes lugares. El campo, igualmente incluiría todos los artefactos necesarios para que tenga lugar la socialización y la posición que adquiera un agente en dicho espacio no solo dependerá de sus reglas internas sino también del campo. En ese sentido, se podría hablar de una realidad social del micro-mundo parcialmente objetiva, porque estaría en función del agente pero también de su entorno y la tecnología. Comenta Sun (2001, p.12) al respecto: 
Aunque la influencia de las sociedades a los individuos es abrumadora, la influencia en la otra dirección puede no obstante discernirse. Tal como subrayan los sociólogos fenomenológicos, la realidad social es un "logro continuo" construido activamente por los agentes a través de prácticas organizadas de la vida cotidiana. La realidad social es, de alguna manera, un producto emergente de las decisiones, acciones y pensamiento de los agentes individuales, cada uno de los cuales tiene una interacción directa y significativa con su mundo.

Así, el agente humano podría representarse como una relación entre sus reglas y su campo, más que un elemento independiente sumergido en un entorno (Dreyfus, 2003), de manera similar a la idea planteada en la hiper-computación biológica, donde no hay una clara frontera entre el organismo y su ambiente (Maldonado, 2016). Por tanto, precisar una línea divisoria entre el agente humano y su entorno tampoco sería posible.

Los agentes inmersos en un micromundo pueden asumir roles en determinados momentos, pero estos pueden cambiar conforme se establecen nuevas relaciones o los agentes desarrollan una nueva interpretación del micromundo. Como estos cambios se desarrollan en una escala temporal, la estructura del micromundo permanecerá estable durante un intervalo de tiempo considerable para luego dar paso a una nueva.

Entre los retos que implica un modelado basado en agentes y micro-mundos, se encuentra el de lograr una auto-interpretación de parte del agente humano. De acuerdo con Heidegger (1927), la auto-interpretación viene dada a través del comportamiento y la interacción directa con el mundo sin que haya una mediación; por tal razón, el enfoque tradicional de la inteligencia artificial, donde el agente tiene una representación del conocimiento de sus deseos o intenciones expresada en un tipo de lógica y realiza inferencias de forma deliberada, debería reformularse (Sun, 2001). En este caso el agente y el micro-mundo deberían enlazarse en un bucle de auto-modificación, donde el actuar del agente se modificaría de manera adaptativa a la medida que el micro-mundo evolucione, según las perturbaciones inducidas por el agente mismo y los demás agentes. Así, los modelos conectivos de computación con retroalimentación (como las redes neuronales recurrentes) podrían ser los primeros motores de acción en los agentes para luego dar cabida a los modelos lógico-simbólicos (Sun, 2001).

\section{Un caso de aplicación}

Como se había anunciado, en esta sección se presenta un caso de aplicación donde se propone modelar el crimen urbano contra la propiedad como el resultado de la dinámica de un sistema socio-técnico. La ciudad, como soporte donde se produce el fenómeno del crimen, se considera en este modelo como la componente técnica del sistema. La ciudad se comprende así como un agregado de tecnologías de construcción, transporte, comunicación, vivienda, recreación, entre otros, que se dispone de manera planeada o auto-organizada para el desarrollo de las 
comunidades (Bettencourt, et al., 2010). De otro lado, el componente social del sistema está dado por un conjunto de actores que activan el crimen al tiempo que otro grupo busca inhibirlo. Los actores toman decisiones en función de las oportunidades de activar o inhibir el crimen e interactúan sobre el soporte dado por la ciudad.

Si bien los actores gozan de cierta autonomía, sus decisiones se ven afectadas por la presencia o ausencia de factores asociados al soporte tecnológico de la ciudad. La ciudad condiciona el actuar de los agentes, manifestándose así la interacción entre las fuerzas de activación e inhibición sobre el territorio. En este sentido, las unidades territoriales se entienden como componentes activos del sistema, donde interactúan fuerzas antagonistas (Melgarejo \& Obregón, 2014), y la generación de eventos criminales caracteriza la dinámica que acontece en cada una de estas unidades.

La ciudad se interpretaría como una red de micro-mundos interconectados en los cuales operan determinadas reglas a través de los agentes, por lo que podría pensarse que cada unidad territorial computa un algoritmo de interacción entre las fuerzas de activación e inhibición. De este modo, la agencia humana queda empotrada en el campo del mundo y su expresión se evidencia en términos de reglas de interacción. La relación entre las fuerzas de activación e inhibición en el micro-mundo y el momento se expresa entonces por el siguiente par de reglas:

$$
\begin{aligned}
& \operatorname{Aij}(t)=f(\operatorname{Aij}(t-1), V(A i j(t-1), \operatorname{Pij}(t-1)) \\
& P i j(t)=g(P i j(t-1), V(P i j(t-1)), \operatorname{Pij}(t-1))
\end{aligned}
$$

Donde $f$ es la regla de activación, $g$ es la regla de inhibición y V(micromundo) hace referencia a los micromundos que rodean al micromundo (i,j) (por ejemplo vecindad). Las fuerzas de activación e inhibición se expresan como masas normalizadas en el intervalo $[0,1]$.

Las reglas $f$ y $g$ son construidas por medio de una lógica no-clásica (Maldonado, 2016) que se soporta sobre un álgebra de De Morgan, que integra los siguientes elementos: $\{0,1,[0,1]$, conjunción, disyunción y negación\} (Walker \& Walker, 2005). Las expresiones lógicas se obtienen por medio de un algoritmo de búsqueda poblacional que minimiza un funcional que mide la similitud entre la dinámica del modelo y patrones tomados de una base de datos de ofensas criminales (por ejemplo hurto a casas) reportadas en la ciudad San Francisco (USA).

A manera de ejemplo, en la Figura 1 se muestran dos patrones generados por el modelo y su comparación con la base de datos para dos días. En ambos casos se puede apreciar la actividad criminal en los micro-mundos representada en una escala de colores. Los colores más claros representan una mayor actividad criminal o mayores niveles de activación Figura 1. Se puede notar una semejanza en la geometría del comportamiento emergente tanto en las simulaciones como en las observaciones: se revelan zonas de alta concentración de actividad 
criminal a la izquierda y zonas de baja concentración a la derecha del macro-mundo.

La caracterización del macro-mundo se presenta de manera resumida en la Figura 2. Allí se ha empleado una medida de entropía derivada de las propiedades multi-fractales del sistema con el fin de establecer una comparación entre los resultados de la simulación y las observaciones disponibles. Se evidencia que el nivel promedio de las dos secuencias es similar, lo que significa que tanto la simulación como la caracterización comparten un comportamiento dinámico similar en términos de la información que los dos producen en diversas escalas.

Este modelo de micro-mundos interconectados que dan forma a un macro-mundo parte de interacciones soportadas en la lógica. Tales interacciones expresadas a manera de reglas concentran en un solo artefacto tanto la presencia de un soporte tecnológico como de un conjunto de actores que manejan determinadas relaciones. Así, la interacción ser-mundo queda empotrada en un modelo que no diferencia entre los dos, siendo esto un aspecto diferenciador con respecto a los modelos de agentes donde actores y campo de juego están claramente diferenciados. Por otro lado, el hecho de emplear reglas lógicas en este modelo, que son capaces de producir patrones complejos sin la necesidad de involucrar parámetros reales ni términos funcionales, estaría a favor de una interpretación computacional y determinística del sistema socio-técnico.

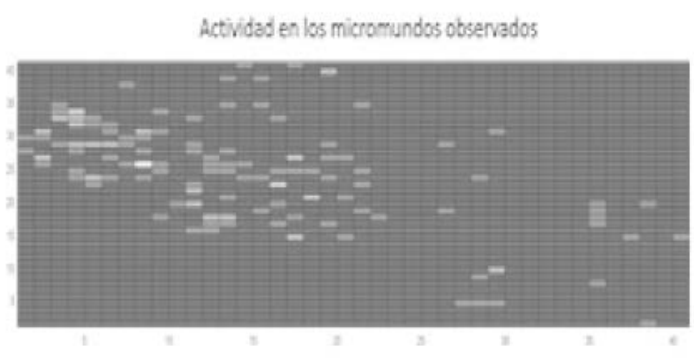

Actividad en los micromundos simulados

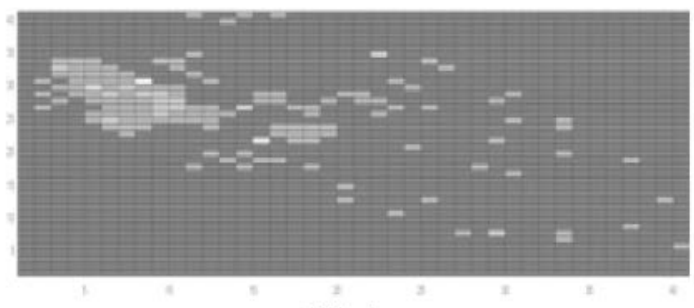

Día 1

Actristad en los miccomuntos obserabos

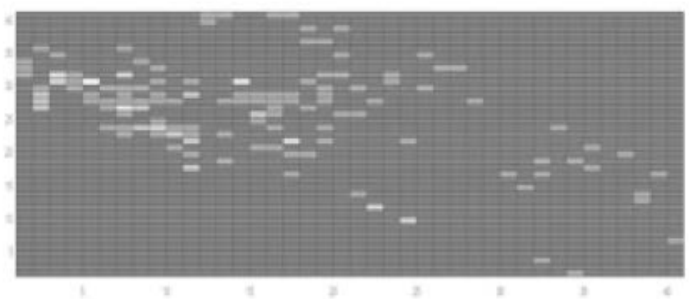

Actividad en los mivomundos simuladsos

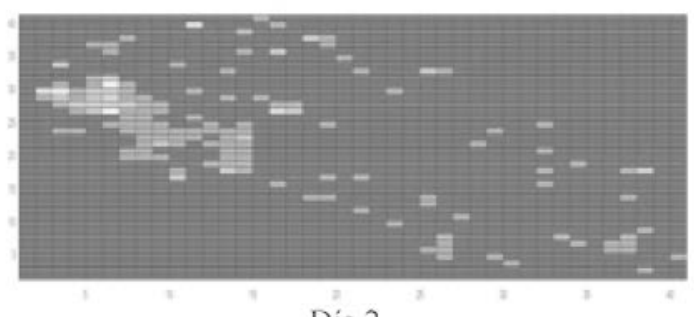

Día 2

Figura 1. Ejemplos de patrones de la actividad criminal en los micro-mundos observados y simulados para los mismos días

Fuente: Elaboración propia 


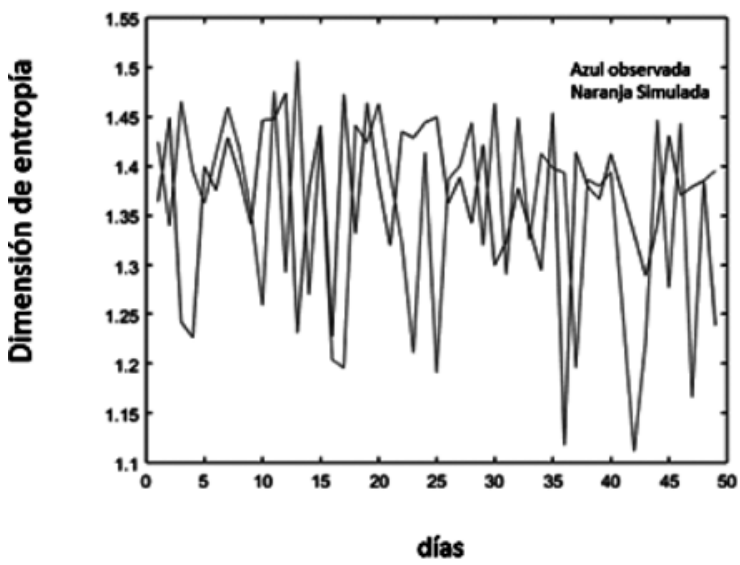

Figura 2. Dinámica resumida en la dimensión de entropía de la actividad criminal en los micro-mundos

Fuente: Elaboración propia

\section{Discusión}

¿El modelamiento de sistemas sociotécnicos debería entenderse como una actividad puramente científica o un campo de interés para la acción? En realidad, si se hablara del sistema socio-técnico por sí mismo, se estaría imponiendo una visión particular de la realidad, dado que las asociaciones y relaciones humanas se estarían entendiendo desde una perspectiva sistémica donde aparecen componentes, flujos de información, materia o energía al igual que enlaces interconectando estos elementos. Esta forma de entendimiento de lo humano y sus colectividades encierra un paradigma de aproximación científica. En tal sentido, dar esta connotación impondría un desocultamiento de lo humano, que se convertiría en un objeto susceptible de ser modelado y representado computacionalmente. Pero al mismo tiempo, esta consideración supondría la necesidad de definir teorías rectoras del comportamiento humano y sus sociedades, quizás moviéndose en contravía de la apertura al ser que caracteriza lo humano.
Aun cuando la aproximación científica encuentra una contradicción al tratar de modelar lo que se ha considerado como un sistema sociotécnico, esto no quiere decir que no se deba formular la pregunta por tales sistemas para tratar de comprender algo sobre su naturaleza. Quizás nunca se podrá controlar la incertidumbre que encierra la comprensión de estos sistemas, sin embargo hacerles frente es una posibilidad. En este caso, la acción se convertiría en una invitación al diálogo y la reflexión sobre el hombre y sus asociaciones desde diferentes visiones mundo. Particularmente, la ingeniería puede aportar un elemento interesante a la discusión, entregando tecnologías de simulación que permitan visualizar diferentes escenarios de comportamiento. La actividad de modelamiento se convertiría entonces en un ejercicio de diseño que debería encontrar una fuente de inspiración en la multiplicidad del conocimiento, el lenguaje y la cultura.

En este trabajo se ha revisado en parte esta última idea respecto a la ingeniería. El modelamiento de un sistema socio-técnico se piensa como una actividad de diseño, entendido como una acción creativa que interconecta fragmentos de virtualidad llamados bloques constructores. La realidad trata de capturarse en una construcción artificial (por ejemplo simulador), no a través de la fragmentación sino de la agregación. Y este proceso de agregación se desarrolla a partir de la necesidad de representar la complejidad subyacente del sistema. Así pues no se busca un modelo general, sino modelos que tengan posibilidad de adaptación al sistema que representan. 
La diferencia que se toca aquí es quizás la figura de bloque constructor, mas no como algo rígido y encajable con otros bloques, sino como un agente activo, que es modificado en la medida que se construyen sus micro-mundos. En cierta forma, el agente lo define, pero a su vez este define al agente. Quizás la misma idea se pueda extrapolar en la construcción del macro-mundo a partir de los micro-mundos: el primero va siendo construido por los segundos, al tiempo que estos son modificados en el proceso de construcción. Un ejercicio mental de la auto-organización de estos mundos de simulación evoca la idea de Heiddeger en cuanto a la mundaneidad: Si uno descubre una estructura común a los sub-mundos de un mundo, habrá encontrado la estructura de este. Sin embargo, la pregunta es si el mundo de simulación de los agentes reflejará en alguna medida el contexto de acción de lo humano.

\section{Conclusión}

Diseñar modelos que traten de capturar la complejidad de un sistema socio-técnico es un gran reto. Esta clase de sistemas exhibe quizás una de las formas más interesantes de complejidad entre los sistemas complejos debido a la presencia del elemento humano. Este factor particular hace que los elementos que componen el sistema realicen una interpretación local y global del mismo, sobre la cual se pueden manifestar procesos de adaptación, auto-organización y evolución. En este contexto, es posible preguntarse por el diseño de sistemas complejos que sirvan de modelos de sistemas socio-técnicos. Desde esta perspectiva, abordar el proceso de diseño implica alejarse de la visión reduccio- nista imperante en la ciencia y en la ingeniería, abriéndose a un campo diferente de formas de diseño basadas en procesos incrementales o evolutivos.

Dado todo lo anterior, es claro que este trabajo presentó una aproximación al diseño de sistemas multi-agentes como artefactos de modelación de sistemas socio-técnicos, desde una perspectiva que integra un proceso de diseño incremental basado en bloques constructores que dan forma a micro-mundos, que a su vez conforman macromundos. Como en la propuesta presentada el bloque constructor es un agente inmerso en el mundo, el comportamiento del agente se interrelaciona con el comportamiento del micro-mundo en el cual se encuentra, por tanto se hablaría de un proceso de adaptación que funcionaría en dos vías: el micro-mundo puede modificar al agente, pero este a su vez puede modificar el micromundo, formando una sola entidad de análisis. Esta aproximación desde la psicología cognitiva podría ayudar a que la simulación de sistemas socio-técnicos se acerque más a la realidad que las propuestas basadas en el uso de agentes objetivamente aislados del mundo que los rodea.

\section{Referencias}

Ackoff, R. \& Gharajedaghi, J. (1996). Reflections on Systems and Their Models. Systems Research and Behavioral Science, 13(1), 13-23.

Arendt, H. (1958). The human condition. Chicago: University Press.

Arnold, T. (2013). Procedural knowledge for 
integrated modeling: towards modeling playground. Environmental modelling \& software, 39(1),135-148.

Bak, P. (1991). Catastrophes and Self-Organized Criticality, Computers in Physics, (5)430433.

Berger, T., Birnerb, R., Díazc, J., McCarthyb N., Wittmerd, H. (2007). Capturing the complexity of water uses and water users within a multi-agent framework. Water Resource Management, (21), 129-148

Bettencourt, L. (2010). Urban scaling and its deviations: Revealing the structure of wealth, innovation and crime across cities. PLoS One, 5(11), 20-22.

Cowan, F., Allen, J., Mistree, F. (2006). Functional Modelling in Engineering Design: a Perspectival Approach Featuring Living Systems Theory. Systems Research and Behavioral Science, 23, 365-381.

Dreyfus, H. (2003). Ser-en-el-mundo comentarios a la división de ser y tiempo de Martin Heidegger. Santiago de Chile Editorial Cuatro Vientos.

Epstein, J. (2008). Why model. Journal of Artificial Societies and Social Simulation, 11(4), http://jasss.soc.surrey. ac.uk/11/4/12.html

Gadamer, H. (1960). Sobre la planeación del futuro. Verdad y Método II, (pp.153-171) Ediciones Salamanca, (Versión en castellano de 1998).

Heidegger, M. (1927). Ser y tiempo. (Traducción Santiago de Chile Escuela de Filosofía, Universidad ARCIS).
Heidegger, M. (1938). La época de la imagen del mundo", Senderos del Bosque. Madrid: Alianza Editorial (Versión castellana 1996).

Jakeman, A., Delden, H., Seppelt, R. \& White, R. (2011). A methodology for the design and development of integrated models for policy support. Enviromental modeling \& software, 26, 266-279.

Jelinek, M., Romme, A. \& Boland, R. (2008). Introduction to the Special Issue. Organization Studies as a Science for Design: Creating Collaborative Artifacts and Research. Organization Studies, 29(3), 317.

Kroes, P. (2012). Engineering Design. In P. Kroes, Ed. Technical Artefacts: Creations of Mind and Matter, (pp.127-161), Springer: Dordrecht.

Kroes, P., Franssen, M., Van de Poel, I., et al. (2006). Treating Socio-Technical Systems as Engineering Systems: Some Conceptual Problems. Systems Research and Behavioral Science, 23, 803-814.

Maldonado, C. (2016). Hipercomputación biológica y comunicación entre los seres vivos. Revista simbiótica, 3(1), 208-229.

Melgarejo, M. \& Obregón, N. (2014). On the design of fuzzy cellular automata following a maximum entropy principle. IEEE Conference on Norbert Wiener in the 21 st Century $(21 \mathrm{CW})$, Boston, 1-5.

Mendel, J. (2007). Computing with words: 
Zadeh, Turing, Popper and Occam, IEEE computational intelligence magazine, 2 (4), 10-17.

Pahl-Wostl, C. (2007). The implications of complexity for integrated resources management. Environmental Modelling \& Software, 22(5), 561-569.

Parrot, L. (2011). Hybrid modeling of complex ecological systems for decision support: recent successes and future perspectives. Ecological informatics, 6, 44-49.

Peñaloza, G. (2015). Una mirada desde la Didáctica de las Ciencias al concepto de visión del mundo. Educación y Humanismo, 17(29), 308-320.

Sice, P. \& French, I. (2006). A Holistic Frame-ofReference for Modeling Social Systems. Kybernetes, 35(6), 851-864.

Simon, H. (1988). The Science of Design: Creating the Artificial. Design Issues, 4(1-2), 67-82.

Schwaninger, M., Olaya, C., Ambroz, K. (2007). The Complexity Challenge: A Case for Model-Based Management. Proceedings of the 25th International Conference of the
System Dynamics Society. Massachusetts. Institute of Technology-System Dynamics Group, Boston, MA, USA.

Sun, R. (2001). Cognitive Science Meets MultiAgent Systems: A Prolegomenon. Philosophical Psychology, 14(1), 5-28.

Torrens, P. M. \& Nara A. (2007). Modeling gentrification dynamics: A hybrid approach. Computers, Environment and Urban Systems, 31, 337-361.

Walker, C. \& Walker, E. (2005). The algebra of fuzzy truth values. Fuzzy sets and systems, 149, 309-347.

Zevski, G. (2012). Modelling Large Complex Systems Using Multi-agent Technology. Proceedings of the 13th ACIS International Conference on Software Engineering, Artificial Intelligence, Networking and Parallel \& Distributed Computing (SNPD), 434-437. 